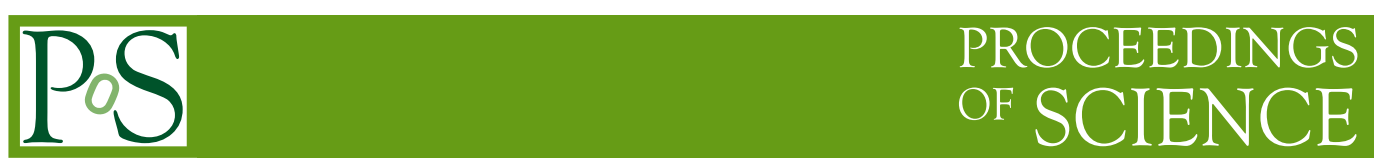

\title{
Review on the LHC Run2 results
}

\section{Yu Nakahama*}

KMI, Nagoya University, Japan

E-mail: yu.nakahama@cern.ch

The second period of the Large Hadron Collider (Run2) started in 2015, producing proton-proton collisions at an unprecedented centre-of-mass energy of $13 \mathrm{TeV}$. During 2015 and 2016, the ATLAS and CMS detectors both collected approximately $40 \mathrm{fb}^{-1}$ of dataset at the new collision energy. In the following proceedings, we briefly introduce to the detector status and performances, and review the recent physics progresses made by the LHC experiments, regarding the Standard Model, the Higgs scalar sector and searches for new particles. The review mainly focuses on the ATLAS experiment that KMI has been heavily contributing to.

The 3rd International Symposium on Quest for the Origin of Particles and the Universe" 5-7 January 2017

Nagoya University, Japan

$$
{ }^{*} \text { Speaker. }
$$




\section{Introduction}

\subsection{The Large Hadron Collider and the ATLAS experiment}

The Large Hadron Collider (LHC) is an energy-frontier collider at CERN, delivering protonproton collision events to two multi-purpose detectors; ATLAS and CMS to dedicated detectors; $\mathrm{LHCb}$ for $B$-physics studies and ALICE for heavy-ion studies. Regarding recent history and future schedule, the LHC first period Run1 (2009-2012) with $p-p$ collisions at the centre-of-mass energy $\sqrt{s}=7-8 \mathrm{TeV}$ The current Run 2 is in a high-energy phase at $\sqrt{s}=13-14 \mathrm{TeV}$ (2015-2018), followed by a bit higher luminosity phase Run3 (2021-2023). The following proceedings review recent physics highlights in Run2. For future, High-Luminosity LHC (2026-) and FCC (far beyond) are planned. The further details can be found in the two other proceedings of KMI2017; on the future physics program by B. Petersen and on muon trigger upgrade by M. Tomoto as one of KMI contributions.

The ATLAS detector is a multi-purpose detector with a forward-backward symmetric cylindrical geometry and nearly $4 \pi$ coverage in solid angle. It is composed of the Inner Detector, the Calorimeters, and the Muon Detectors, from the inner to the outer layers, as well as of the Trigger and Data acquisition system. It can directly explore energy scale from $O(10) \mathrm{GeV}$ to $O(10) \mathrm{TeV}$. As of 2017 January, the ATLAS experiment has around 2900 collaborators from 180 institutions across 38 countries. KMI has been contributing to the ATLAS experiment with primary focuses on Muon Detector and Trigger system, as well as on Top physics and searches for New Physics beyond the Standard Model (SM).

As physics highlights in Run1, we should note the discovery of the Higgs Boson; Higgs-like particle discovery was announced on July 4th 2012. In March 2013, key papers were published on the properties of the new particle, which has been declared as a Higgs boson. These papers [1] were cited in the description for the 2013 Nobel Prize in Physics. The other important highlight to be mentioned is a deep and broad range of experimental results, all beautifully fitting in the SM framework of the elementary particle physics.

\subsection{Experimental Status of the ATLAS Run2 Period}

Primary physics focuses in Run2 are (1) on New Physics searches using early dataset and (2) on measurements of Higgs properties using higher-statistics dataset.

For Run2, new sub-detectors installed in the ATLAS detector; Innermost pixel layer (IBL) placed at $3.4 \mathrm{~cm}$ away from the interaction point and Forward proton detectors (AFP) at $210 \mathrm{~m}$ from Interaction Point.

In addition, various consolidations provide improved running at high luminosity and rates expected in Run2; for instance in triggers. Here, KMI has been giving critical contribution including menu-coordination role. We designed and operated a complex list of trigger selections to meet varied physics, monitoring and performance requirements. There operated typically 2000 active trigger selections, driven by the priorities in the ATLAS physics programme. We kept stable primary triggers with a peak output rate at Level-1 Trigger of $90 \mathrm{kHz}$ and an average output rate at High Level Trigger to storage of $1.0 \mathrm{kHz}$, which fully met the detector operation and data-acquisition requirements 
The $2016 p$ - $p$ data taking lasted until 26th October, setting a record in peak luminosity of $1.4 \times 10^{34} \mathrm{~cm}^{-2} \mathrm{~s}^{-1}$. Total dataset recorded was $36 \mathrm{fb}^{-1}$ with excellent data quality with more than 90 percentage of data collected is usable for physics analyses.

\section{Physics Results in the ATLAS Run2 Period}

As of January 2017, the ATLAS experiment has submitted 605 papers in total from varied physics areas, including 51 papers using Run2 dataset. In this section, we review a few selected highlights, mostly using $13 \mathrm{fb}^{-1}$ dataset, collected up to 2016 summer in the LHC Run2.

\subsection{Standard Model Measurements}

Various measurements of the SM processes were performed. In this section, three representative topics are described; Inclusive cross-section measurements of the SM processes, precise measurement of the $W$-boson mass, and measurements of the $t \bar{t}$ cross-sections.

\subsubsection{Inclusive cross-section measurements of the SM processes}

A broad range of inclusive cross-section measurements of the SM processes was performed, summarised in Figure 1, from $p$ - $p$ inelastic scattering to the diboson $Z Z$ production [6]. These many measurements from Run1 and Run2 are important steps forward in physics modeling in the last years; In the Monte Carlo (MC) samples, NLO event generators are currently used as standard. (N)NNLO calculations are increasingly available. These help to face the challenge of the precision of the LHC data. Measuring complex topologies bench-test MC models of the SM backgrounds to New Physics searches.

\subsubsection{Precise measurement of the $W$-boson mass}

Precise measurement of the $W$-boson mass is well motivated for a consistency test of the SM and a probe of BSM physics. After long and huge efforts, the first ATLAS result using the $7 \mathrm{TeV}$ dataset, corresponding to $4.6 \mathrm{fb}^{-1}$ of integrated luminosity, was released in December 2016 [5]; $\mathrm{m}_{W}=80370 \pm 7$ (stat.) \pm 11 (exp. syst.) \pm 14 (mod. syst.) $\mathrm{MeV}=80370 \pm 19 \mathrm{MeV}$. This value is competitive to the previous results by the LEP and the Tevatron, and is dominated by physics modeling uncertainties as expected. The result is used for consistency tests of the SM using the Higgs mass and the top-quark mass, and shows no deviations from the SM expectations in the global electroweak fit.

\subsubsection{Measurements of the $t \bar{t}$ cross-sections}

Precise measurements of inclusive $t \bar{t}$ cross-sections were performed using the $13 \mathrm{TeV}$ dataset, corresponding to $3.2 \mathrm{fb}^{-1}$ of integrated luminosity. Measurement precision $\pm(3.9-4.4)$ is better than theory NNLO+NNLL predictions (5\%). Further high statistics allow detailed studies of production properties, for instance, differential cross-section measurements. 


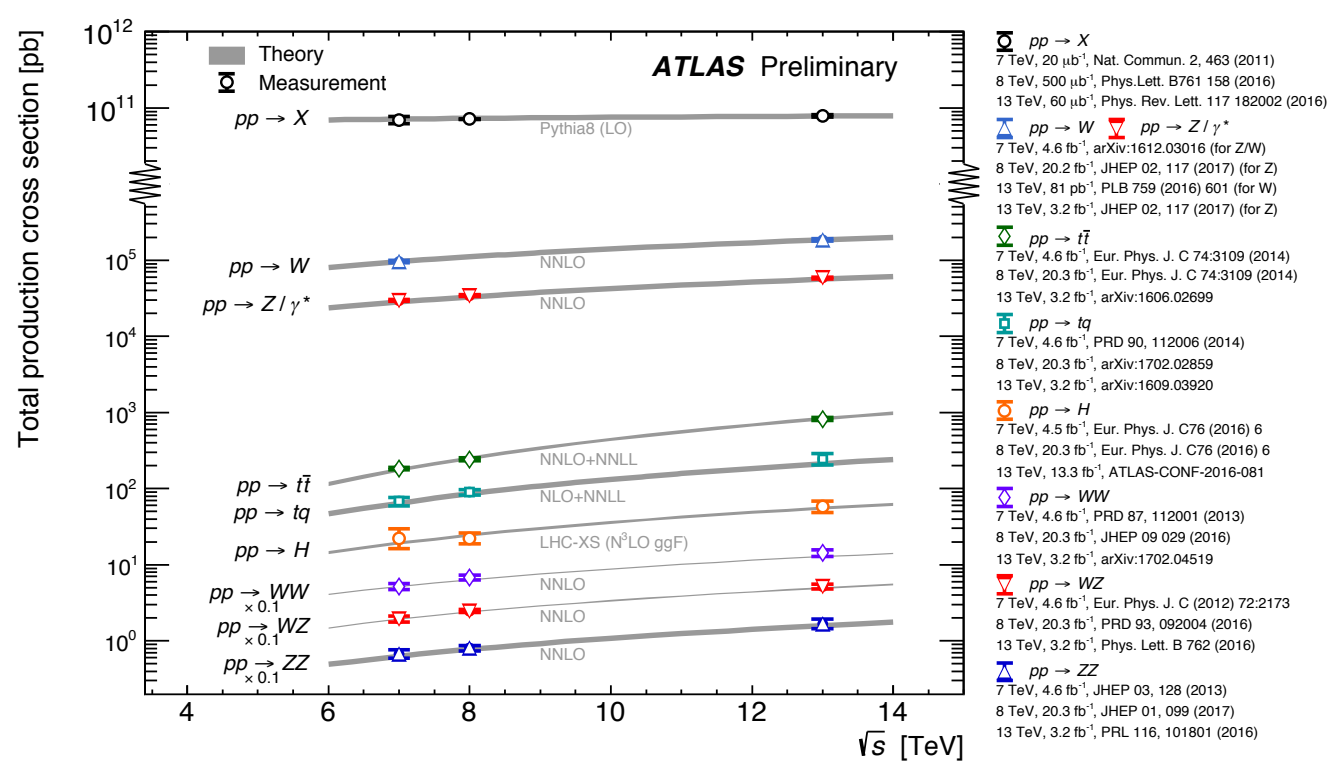

Figure 1: Summary of total production cross-section measurements by ATLAS presented as a function of centre-of-mass energy $\sqrt{s}$ from 7 to $13 \mathrm{TeV}$ for a few selected processes. The diboson measurements are scaled by a factor 0.1 to allow a presentation without overlaps.

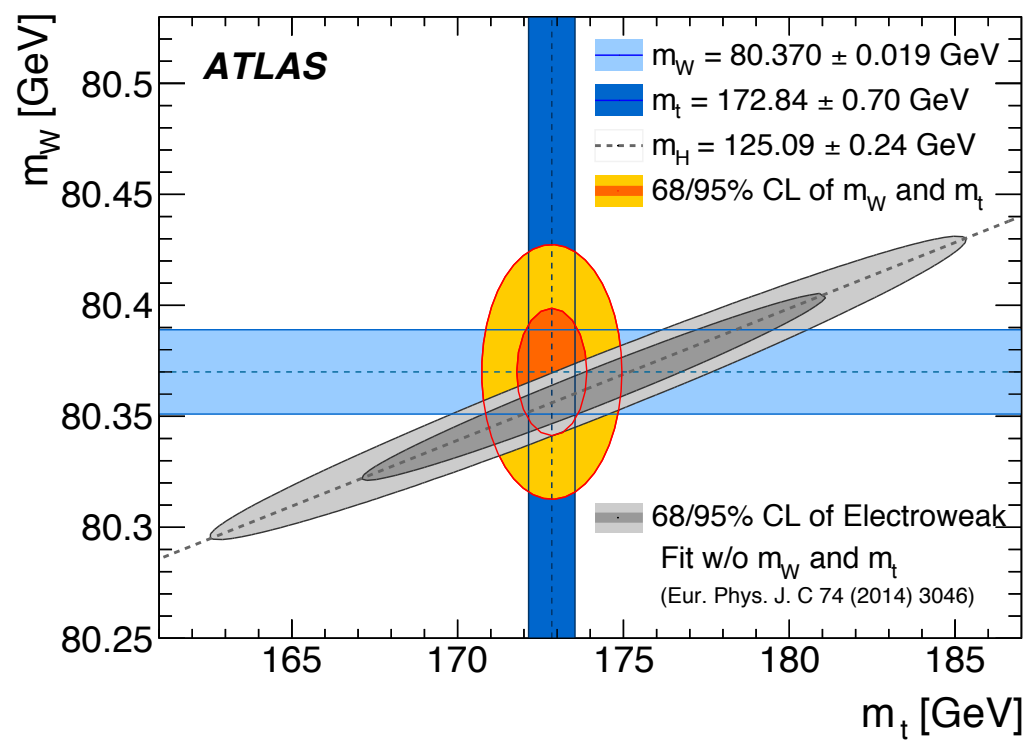

Figure 2: The $68 \%$ and $95 \%$ confidence-level contours of the $\mathrm{m}_{W}$ and $\mathrm{m}_{t}$ indirect determination from the global electroweak fit are compared to the $68 \%$ and $95 \%$ confidence-level contours of the ATLAS measurements of the top-quark and $W$-boson masses. The determination from the electroweak fit uses as input the LHC measurement of the Higgs-boson mass, $\mathrm{m}_{H}=125.09 \pm 0.24 \mathrm{GeV}$. 


\subsection{Higgs Physics}

Higgs discovery opened the door on the scalar sector. In Run1, we measured: its spin-parity, and its mass precisely $( \pm 0.2 \%)$, production via gluon-fusion, vector-boson fusion, and with a $W$ or $Z$, the bosonic decays to $\gamma \gamma, W W, Z Z$, and the fermionic decay to $\tau \tau$. These proved true the BEH mechanism to break gauge symmetry. However, apart from the mass, none of these measurements are yet very precise and leave room for significant deviations. Also, important modes are still unseen $(b b, t t H, \mu \mu, Z \gamma, H H)$. Run2 priorities are: establish and measure at $13 \mathrm{TeV}$, search for $t t H$ production to probe $t t H$ Yukawa coupling directly, search for $H \rightarrow b b$ decays, search for rare decays, Refine measurements of couplings, mass, etc and expand use of the Higgs boson as a tool to find New Physics.

\subsubsection{The Bosonic-decay Channel of the Higgs boson}

With the 2015 dataset at $\sqrt{s}=13 \mathrm{TeV}$, we see clear re-observation of the Higgs decays in bosonic channels: $H \rightarrow \gamma \gamma$ and $H \rightarrow Z Z^{*} \rightarrow 4 \ell$.

\subsubsection{The $t t H$-production Channel of the Higgs boson}

This measurement of $t t H$-production channel allows direct measurement of Higgs-top coupling. CMS performed the first Run2 search for $t t H$ production using a few $\mathrm{fb}^{-1}$ dataset at 13 $\mathrm{TeV}$ in all major channels (multi-leptons, $b b$ and $\gamma \gamma$ ) in March 2016 for Moriond 2016. This requires highly complex analyses, huge effort to get these done so quickly after data taking. ATLAS showed the preliminary result of measurements of signal strength using higher-stat dataset (around $13 \mathrm{fb}^{-1}$ at $13 \mathrm{TeV}$ ) in all major channels and their combination in August 2016 at ICHEP 2016. As summarised in Figure 3, this shows observed significance $2.8 \sigma$ (expect $1.8 \sigma$, showing improved sensitivity over Run1) [4].

\subsubsection{Searches for New physics with the Higgs boson as a tool}

Higgs sector may be non-minimal and/or Higgs boson may couple to New Physics. Diverse search program is in place but no deviations from the SM expectations are found in any of these searches, for instance in $H \rightarrow \mu \mu$ decays and $H H$ production, whose SM processes are expected to be observed using the high-statistics dataset in Run3 and High-Luminosity LHC.

\subsection{Searches for New Physics Phenomena; Exotics and Supersymmetry}

Broad search program is performed from inclusive surveys of basic event topologies to dedicated searches ruling out corners of phase space. Thanks to the centre-of-mass-energy increase in Run2 from $8 \mathrm{TeV}$ to $13 \mathrm{TeV}$, major extension of reach is obtained compared to Run1. All results shown in this section include the $13 \mathrm{TeV}$ dataset collected in 2016. They probe well into the $\mathrm{TeV}$, even multi-TeV, mass scale range in searches for New Physics phenomena; Exotics and Supersymmetry (SUSY).

\subsubsection{Exotics}

The defined program is to search as broad and general as possible (signatures, masses, rates) covering physics without guiding models. Some interesting excesses were seen in some of the searches and pursued with higher statistics dataset in 2015 and 2016. 


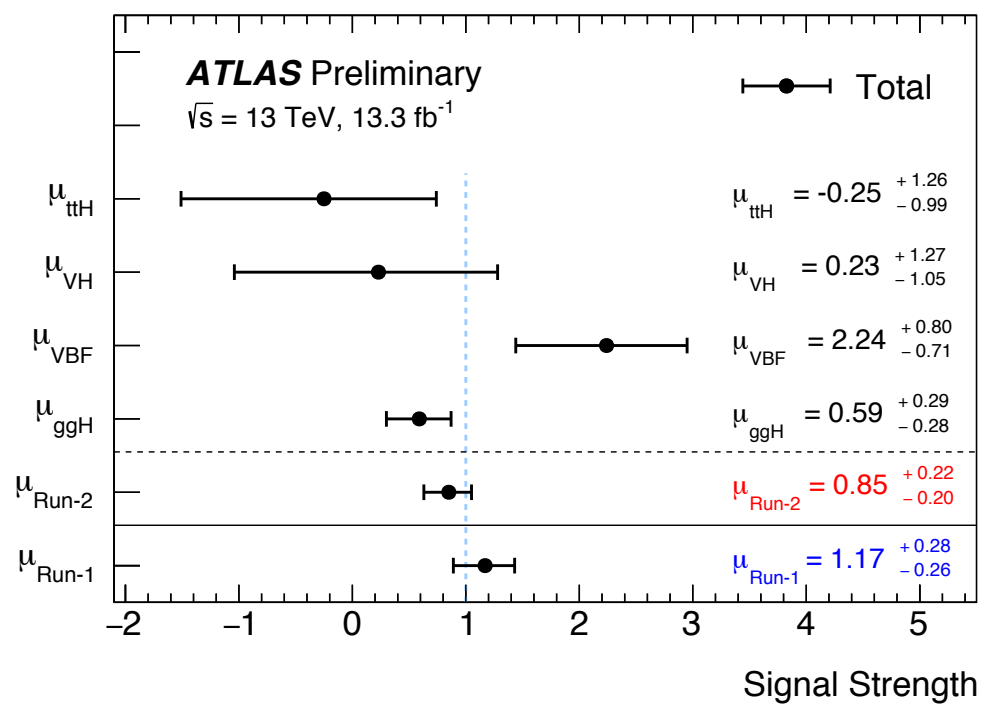

Figure 3: Summary of the observed $t t H$ signal strength measurements from the individual analyses and for their combination, assuming $\mathrm{m}_{H}=125 \mathrm{GeV}$. The total (tot.), statistical (stat.), and systematic (syst.) uncertainties on $t t H$ are shown. The SM $\mu_{t t H}=1(0)$ expectation is shown as the black (grey) vertical line. The observed $t t H$ signal strength measurement obtained from the Run1 combination is also shown for comparison (bottom).

Diphoton search was performed using the 2015 dataset and showed localised excess with 2.1 $\sigma$ global $(3.9 \sigma$ local) significance at $750 \mathrm{GeV}$ (spin-0 search), width $50 \mathrm{GeV}$. When we added more statistics dataset in 2016 (with 3.8 times more statistics), there is no clustering around 730$750 \mathrm{GeV}$ [3]. The 2016 data is consistent with 2015 at $2.7 \sigma$. This appears that the 2015 excess is a statistical fluctuation. Finally with the 2015 and 2016 dataset, there exists small excess at 710 $\mathrm{GeV}$ but less significance (i.e. $1.4 \sigma$ local, less than $1 \sigma$ global).

Massive diboson search was performed using boosted ( $f a t$ jet) selections (vital at high- $p_{\mathrm{T}}$ ), targeting for instance a new heavy vector boson $\mathrm{W}$ prime, and further explored in many channels with the 2016 dataset [7]. As in Figure 4, overlaying limits are obtained from all the $W Z$ searches performed at $\sqrt{s}=13 \mathrm{TeV}$. There are no persistent excesses, in contrary to the excesses seen in the Run1 dataset.

Search for New Physics was performed also in dijet mass spectrum as bump hunter, as one of the high-priority analysis in early Run2. Sensitivity is greatly extended based on signal shapes even including large low mass tails. This measurement can be utilised for Dark-Matter interpretation, giving a complementary to mono- $\mathrm{X}$ searches, and cover a broad mediator mass range.

\subsubsection{SUSY}

The case for SUSY is well known; a natural candidate of Dark Matter in neutralino1, and can solve the fine-tuning problem, if top squarks $(\tilde{t})$ and gluinos $(\tilde{g})$ are light. In Run1 we identified: $\mathrm{m}_{\tilde{g}}$ is larger than $1.4 \mathrm{TeV}$ for low-mass neutralino1 and $\mathrm{m}_{\tilde{t}}$ is larger than $700 \mathrm{GeV}$ if neutralino1 is lighter than $100 \mathrm{GeV}$. 


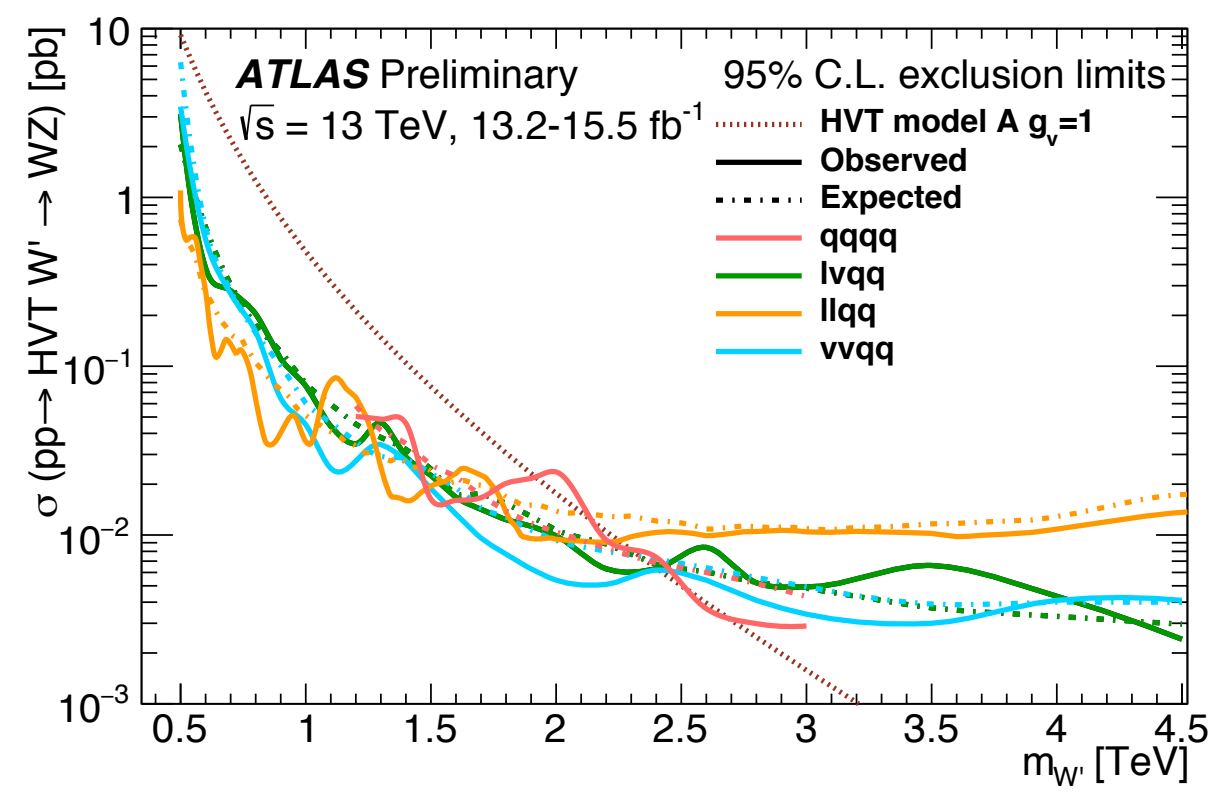

Figure 4: Expected and observed limits on the cross section times branching fraction to $W Z$ for a new heavy vector boson $\mathrm{W}$ prime at $\sqrt{s}=13 \mathrm{TeV}$. The different limit curves correspond to different decay modes for the $W$ and $Z$ bosons.

Run2 priorities are to extend mass reach according to $\sqrt{s}$ increase (e.g. $\tilde{g}$ pair production [8]), to fill "kinematic holes" where particles are soft (e.g. $\tilde{t}$ pair production where the masses of SUSY particles are compressed [9]), and leave no stone unturned e.g. in displaced decays from long-lived particles.

Figure 5 summarises the mass reach on the SUSY particles from the various ATLAS experiment [10]. In brief, huge number of signature-based searches for various production and decay processes within SUSY, in many final states, providing stringent limits under various assumptions; Many of these limits reach around $1 \mathrm{TeV}$ in easy inclusive signatures using the Run2 data. More searches will come for smaller-cross section processes (EW) or for difficult signatures (Long-lived particles).

\section{Summary}

The LHC is performing extremely well. A broad range of LHC Run2 results using early 2016 dataset is available. Regarding the Higgs physics, we start precise measurements of the SM Higgs boson at $\sqrt{s}=13 \mathrm{TeV}$. We conclude on the SM $t$ tH sensitivity. The energy increase from $8 \mathrm{TeV}$ to $13 \mathrm{TeV}$ in Run2 brings us significant increase in sensitivity, shaping the wide search program in 2015 and early 2016. The limits set significantly extend the Run1 results, thus further constraining various models. There are no significant excesses, though some $3 \sigma$ effects exist as expected. More data will tell which, if any, will remain. The search program is moving toward more challenging signatures and scenarios. 


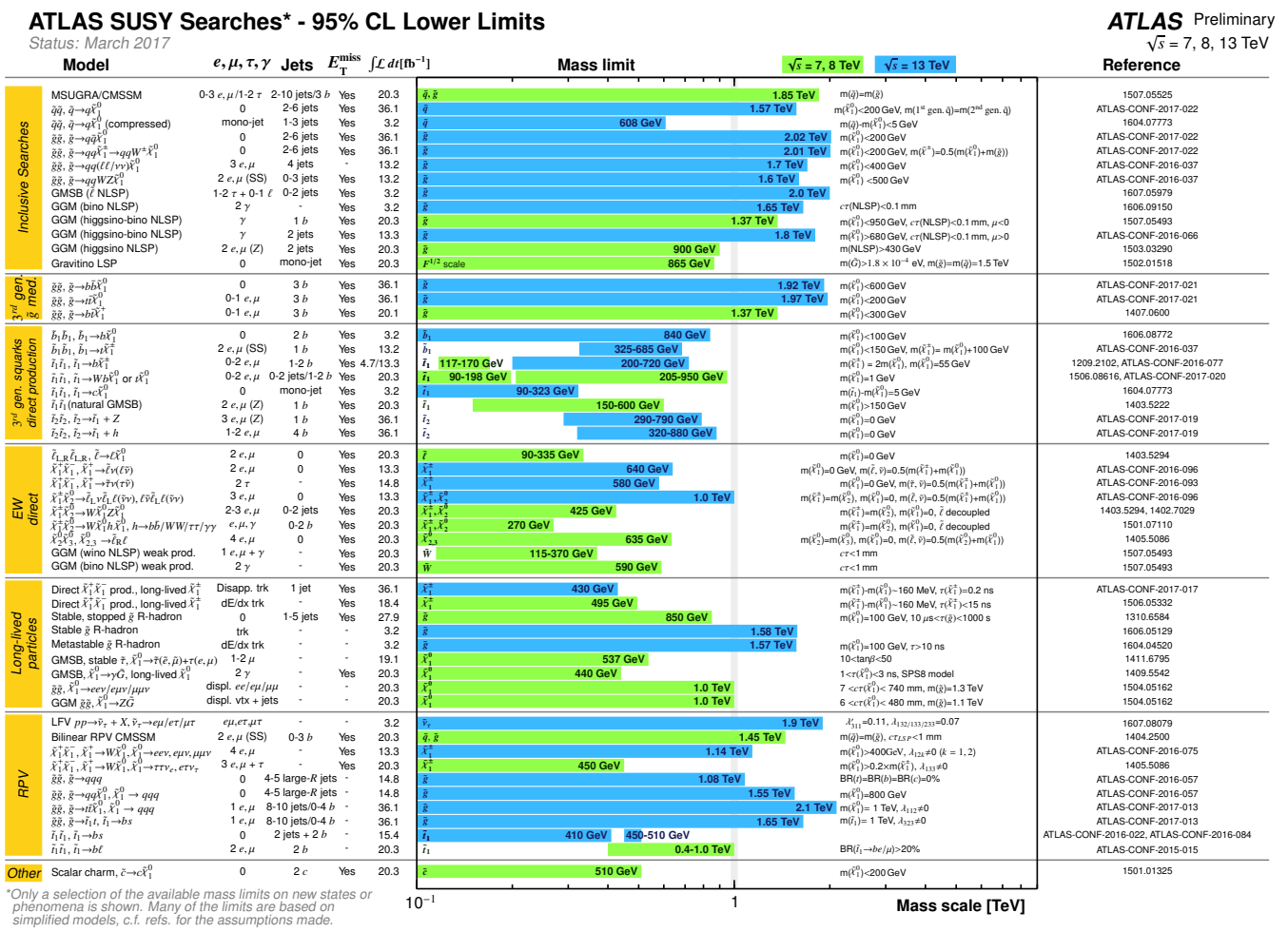

Figure 5: Mass reach of ATLAS searches for SUSY. Only a representative selection of the available results is shown.

We expect exciting future with many new results with $40 \mathrm{fb}-1$ in early spring of 2017 . Data analysed so far is a small fraction of total Run2 dataset (expecting to collect $150 \mathrm{fb}^{-1}$ ).

\section{References}

[1] Observation of a New Particle in the Search for the Standard Model Higgs Boson with the ATLAS Detector at the LHC, the ATLAS collaboration, Phys. Lett. B 716 (2012) 1-29. Measurements of Higgs boson production and couplings in diboson final states with the ATLAS detector at the LHC, the ATLAS collaboration, Phys. Lett. B 726 (2013), pp. 88-119. Evidence for the spin-0 nature of the Higgs boson using ATLAS data, the ATLAS collaboration, Phys. Lett. B 726 (2013), pp. 120-144.

[2] Summary plots from the ATLAS Top physics group, the ATLAS collaboration, https://atlas.web.cern.ch/Atlas/GROUPS/PHYSICS/CombinedSummaryPlots/TOP/

[3] Search for scalar diphoton resonances with $15.4 \mathrm{fb}^{-1}$ of data collected at $\sqrt{s}=13 \mathrm{TeV}$ in 2015 and 2016 with the ATLAS detector, the ATLAS collaboration, ATLAS-CONF-2016-059

[4] Combination of the searches for Higgs boson production in association with top quarks in the $\gamma \gamma$, multilepton, and $b \bar{b}$ decay channels at $\sqrt{s}=13 \mathrm{TeV}$ with the ATLAS Detector, the ATLAS collaboration, ATLAS-CONF-2016-068

[5] Measurement of the W-boson mass in pp collisions at $\sqrt{s}=7 \mathrm{TeV}$ with the ATLAS detector, the ATLAS collaboration, https://arxiv.org/pdf/1701.07240.pdf, submitted to EPJC 
[6] Summary plots from the ATLAS Standard Model physics group, the ATLAS collaboration, https://atlas.web.cern.ch/Atlas/GROUPS/PHYSICS/CombinedSummaryPlots/SM/

[7] Summary plots from the ATLAS Exotic physics group, the ATLAS collaboration, https://atlas.web.cern.ch/Atlas/GROUPS/PHYSICS/CombinedSummaryPlots/EXOTICS/index.html

[8] Search for squarks and gluinos in final states with jets and missing transverse momentum using 36 $\mathrm{fb}^{-1}$ of $\sqrt{s}=13 \mathrm{TeV}$ pp collision data with the ATLAS detector, the ATLAS collaboration, ATLAS-CONF-2017-022

[9] Search for top squarks in final states with one isolated lepton, jets, and missing transverse momentum in $\sqrt{s}=13 \mathrm{TeV}$ pp collisions with the ATLAS detector, the ATLAS collaboration, ATLAS-CONF-2016-050

[10] Summary plots from the ATLAS Supersymmetry physics group, the ATLAS collaboration, https://atlas.web.cern.ch/Atlas/GROUPS/PHYSICS/CombinedSummaryPlots/SUSY/ 